East African Medical Journal Vol. 85 No. 9 September 2008

PREVALENCE OF ANTI-THYROID ANTIBODIES IN PATIENTS WITH PRIMARY THYROID DISORDERS

J. G. Kuria, MBChB, MMed, Lecturer and A. Amayo, MBChB, MMed, Senior Lecturer, Department of Human Pathology, College of Health Sciences, University of Nairobi, P.O. Box 19676-00202, Nairobi, Kenya

Request for reprints to: Dr. J.G. Kuria, Department of Human Pathology, College of Health Sciences, University of Nairobi, P.O. Box 119-00202, Nairobi, Kenya

\title{
PREVALENCE OF ANTI-THYROID ANTIBODIES IN PATIENTS WITH PRIMARY THYROID DISORDERS
}

\author{
J. G. KURIA and A. AMAYO
}

\begin{abstract}
Objective: To determine prevalence of thyroid antimicrosomal and antithyroglobulin antibodies among patients with primary thyroid disorders.

Design: Descriptive cross-sectional study.

Setting: Kenyatta National Hospital, July 2003 to August 2004.

Results: Antimicrosomal antibodies (anti-TPOAbs) were detected in $51.4 \%$ of all the patients while $36.1 \%$ tested positive for the antithyroglobulin antibodies (anti- TgAbs). Among the hyperthyroid patients, anti-TPOAbs and anti-TgAbs were in $50.8 \%$ and $33 \%$ respectively while $53.3 \%$ and $46.7 \%$ of the hypothyroid patients tested positive for anti-TPOAbs and anti-TgAbs respectively. Most of the patients who showed positivity for anti-TgAbs $(88.5 \%)$ also tested positive for anti-TPOAbs.

Conclusion: More than half of the patients with primary thyroid disorders show presence of autoimmune thyroid markers, with anti TPO antibodies being more prevalent.
\end{abstract}

\section{INTRODUCTION}

The role of autoantibodies in the causation of functional thyroid disorders has been known for a long time (1). Autoimmune thyroiditis was first described by Hashimoto who reported four patients with goitre in whom the thyroid histological appearance manifested among other features diffuse lymphocytic infiltration (2). In areas where there is no iodine deficiency, autoimmunity has become the commonest cause of thyroid disease (1). The two main antibodies implicated in autoimmune thyroid disease are antithyroperoxidase and antithyroglobulin antibodies. These autoantibodies can exert a stimulating or a blocking effect on cell membrane receptors leading to alteration in thyroid gland function.

The reported prevalence of thyroid autoantibodies shows variability depending on the geographical location, gender, age as well as the sensitivity and specificity of the analytical methodology used to determine the autoantibodies. Higher prevalence rates have been reported when sensitive methods for autoantibodies detection are utilised.

Alarge study in the United States involving more than 17,000 subjects found anti-TPOAbs and anti-
TgAbs, in $11.3 \%$ and $10.4 \%$ of euthyroid individuals respectively (3). Most of the patients with thyroid disease had detectable antithyroid antibodies. In another study, anti-TPOAbs and anti-TgAbs were found to be present in $85 \%$ and $30 \%$ to $60 \%$ of patients with primary thyroid diseases respectively (4).

Reports on antithyroid antibodies among Africans are few and mostly emanate from studies using agglutination methods which have low sensitivity and specificity. An earlier local study reported anti-TPOAbs and anti-TgAbsin $54 \%$ and $12 \%$ of patients with thyrotoxicosis respectively (5). More recently, Okosieme et al, (6) using a sensitive ELISA technique, reported presence of anti-TgAbs and antiTPOAbs in $11.6 \%$ and $76.8 \%$ of Nigerian patients with Graves disease. This study sought to determine the prevalence of these thyroid antibodies among patients with primary hyper- and hypothyroidism, using the more sensitive assays that have been developed.

\section{MATERIALS AND METHODS}

This was a descriptive cross-sectional study carried out at the Kenyatta National Hospital, Nairobi. Between July 2003 and August 2004 serum samples from patients aged 15 to 60 years with primary hyperthyroidism and hypothyroidism were tested 
for the presence of anti-TPOAbs and anti-TgAbs. Approval for the study was obtained from the KNH-Ethical Research Committee (KNH-ERC) and all patients gave informed consent. Patients with secondary causes of thyroid dysfunction such as pituitary disease and pregnant women were excluded from the study. Determination of antithyroid antibodies was done using an ELISA method with commercial reagents. Results were reported in index values. Index values $>1.10$ were considered positive.

\section{RESULTS}

Out of the 72 subjects with primary thyroid disease, 57had hyperthyroidism and 15 had hypothyroidism. Most of the patients (80.6\%) werefemales and majority (70.8\%) were in the 31-50 years age group. AntiTPOAbs and anti-TgAbs antibodies were detected in $51.3 \%$ and $36.1 \%$ of the patients respectively (Figure 1).

\section{Figure 1}

Age related distribution of anti $-\mathrm{Tg} A$ Abs seropositivity

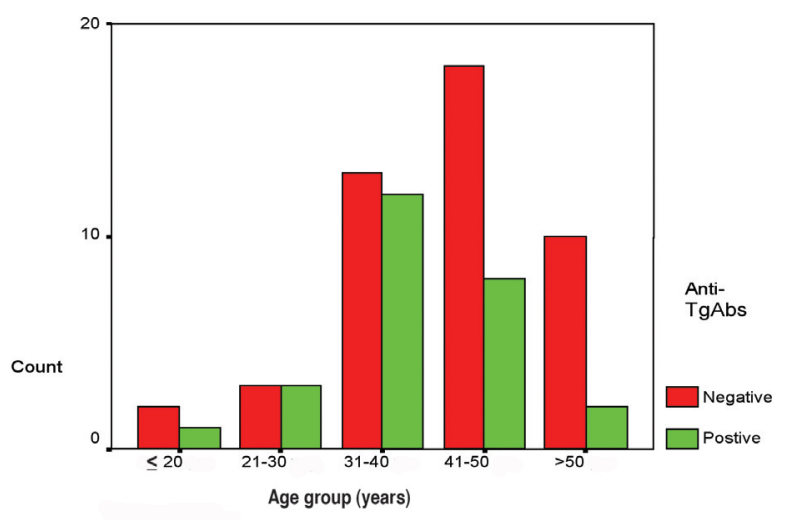

Figure 2

Age related distribution of anti-TPOAbs seropositivity

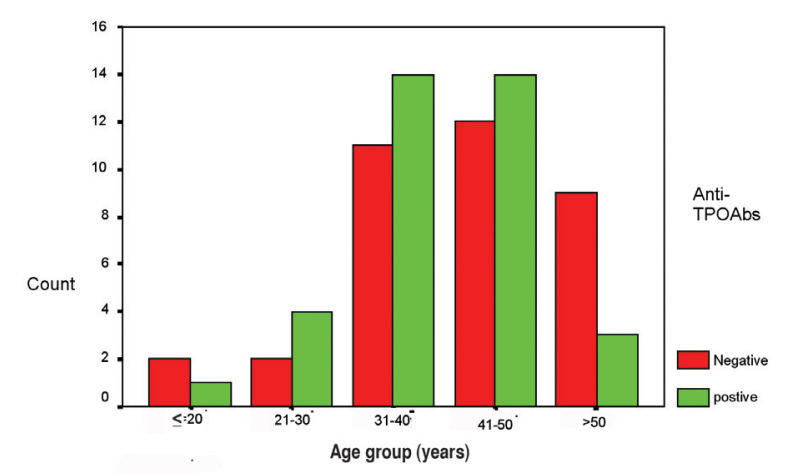

Twenty nine $(50 \%)$ of the female patients tested positive for anti-TPOAbs while $18(31 \%)$ of the females tested positive for anti-TgAbs. Eight males tested positive for each of the antibodies (Table 1). Most of the patients who were positive for anti-TPOAbs $(88.5 \%)$ were also positive for anti-TgAbs. Among the patients who tested positive for the antibodies, levels of anti-TPOAbs ranged from 21 to $688 \mathrm{IU}$ while those of anti-TgAbs ranged from 20 to $330 \mathrm{IU}$.

\section{DISCUSSION}

Autoimmune thyroid diseases are among the most common autoimmune disorders in man. It has been reported that thyroid autoimmunity is rare in iodine deficient areas but becomes more prevalent with improvement in iodine nutrition (6-8). Locally, it is a requirement that all domestic salt is iodinated in an attempt to reduce the occurrence of iodine deficiency disorders (9). The presence of antimicrosomal antibodies (anti-TPOAbs) is the hallmark of disease

Table 1

Seropositivity to antithyroid antibodies in patients with primary thyroid disorders

\begin{tabular}{|c|c|c|c|c|c|c|c|c|c|}
\hline & & \multicolumn{2}{|c|}{$\begin{array}{c}\text { Anti- TgAbs } \\
\text { Positive }\end{array}$} & \multicolumn{2}{|c|}{$\begin{array}{l}\text { Anti-TPOAbs } \\
\text { Positive }\end{array}$} & \multicolumn{2}{|c|}{$\begin{array}{l}\text { Positive for } \\
\text { both Abs }\end{array}$} & \multicolumn{2}{|c|}{$\begin{array}{c}\text { Negative for } \\
\text { both Abs }\end{array}$} \\
\hline & & No. & $(\%)$ & No. & $(\%)$ & No. & $(\%)$ & No. & $(\%)$ \\
\hline \multirow[t]{2}{*}{ Hyperthyroid } & Males & 6 & 23.1 & 6 & 16.2 & 5 & 21.7 & 3 & 9.4 \\
\hline & Females & 13 & 50 & 23 & 62.2 & 12 & 52.2 & 23 & 71.9 \\
\hline \multirow[t]{3}{*}{ Hypothyroid } & Males & 2 & 7.7 & 2 & 5.4 & 2 & 8.7 & 2 & 6.2 \\
\hline & Females & 5 & 19.2 & 6 & 16.2 & 4 & 17.4 & 4 & 12.5 \\
\hline & Total & 26 & 100 & 37 & 100 & 23 & 100 & 32 & 100 \\
\hline
\end{tabular}


activity (10). It is generally known that females suffer more from autoimmune thyroid disorders than males (11). In this study, $80.6 \%$ patients were females. This compares with the study from Nigeria which found $77.9 \%$ of patients with thyroid disorders being females (6).

Fifty seven per-cent of the males and 50\% of the female patients studied tested positive for anti-TPOAbs. This is similar to a report from Asia where $59 \%$ of the male and $51.67 \%$ of the female patients studied tested positive for the antibody (12). As in this study, most of the patients in the study were in the 30 to 50 year age group. In a recent study among 104 patients with thyroid disorders in Nigeria, anti-TPOAbs were found in $60.5 \%$ of the patients (6).

Among the hyperthyroid patients in this study, 51.3\% tested positive for anti-TPOAbs. This compares with the $54 \%$ found to have the antibodies in an earlier study done locally using haemagglutination method (5). In contrast, $36.1 \%$ of the hyperthyroid patients showed positivity to anti-TgAbs which is higher than what had been reported in previous studies done locally $(5,13)$.

The difference in the positivity rate for anti$\mathrm{TgAbs}$ between these studies is likely to be a reflection of the differences in sensitivities of the methods used. Indirect immunofluorescence and haemagglutination methods, both of which are less sensitive than the ELISA method used in the current study were used in the previous studies. It may also reflect an increase in the immunological burden due to the high level of environmental pollution and many parasitic and infectious diseases leading to increased production of autoantibodies to various body tissues including the thyroid (14).

Previous reports indicate that anti-TgAbs occur more frequently in females than in males (3). A higher proportion of hyperthyroid males $(60 \%)$ showed positivity for the antibody than hyperthyroid females $(27.6 \%)$ in the current study. However, the number of hyperthyroid malesin this study was small $(n=10)$, and may therefore not be representative of the population. Among the hypothyroid patients, antithyroid antibodies were detected in $53.3 \%$. These were mainly anti-TPOAbs with only a few cases showing reactivity to anti- $\mathrm{TgAbs}$ alone.

Most of the patients $(88.5 \%)$ who tested positive for anti-TPOAbs were also positive for the antiTgAbs antibodies. On the other hand, only $62.7 \%$ of those who were positive for anti-TPOAbs tested positive for anti-TgAbs. This finding is similar to what is reported in the literature where up to $100 \%$ of patients with anti-TgAbs tested positive for anti-TPOAbs $(1,4)$. This may be because the two antibodies are polyclonal (15). Again, the number of epitopes in the anti-TgAbs is large as seen in some of the antisera raised from hyperimmunised animals showing up to 40 epitopes (16), and the two antigens have been shown to share some common epitopes (17).

It is also known that when these two antibodies are present, the anti-TPOAbs titres are usually higher $(1,5)$. In this study, the mean titres of anti-TPOAbs were higher than those of anti-TgAbs. Also, patients who tested positive for both antibodies tended to have higher titres of anti-TPOAbs than of anti-TgAbs. In resource poor settings, it may therefore be cost effective to only measure the levels of anti-TPOAbs as a surrogate marker for autoimmunity. No association was however found between the titres of anti-TgAbs or anti-TPOAbs and the severity of thyroid hormone disturbance. This is similar to what was reported in an earlier study (3).

Studies done using modern assay techniques have shown that anti-TPOAbs develop in almost all patients with autoimmune thyroid disease during some stage in the disease process (10). AntiTPOAbs are now considered as a more important marker for autoimmune thyroid disease than anti-TgAbs $(12,18)$. In thyrotoxic patients with autoimmune thyroid disease, titres of anti-TPOAbs tend to fall once they become euthyroid (19). This also happens among hypothyroid patients who are treated with thyroxine. It has been shown that patients with Grave's disease who have high titres of anti- $\mathrm{TgAbs}$ and / or anti-TPOAbs are more likely to become hypothyroid later (20). Medical treatment is the mainstay of treatment of patients with Grave's disease but surgery is indicated in patients with large symptomatic goiters and those intolerant to antithyroid medication. Surgery is also recommended in those with failure to respond to antithyroid drugs and those with nodules which are suspicious for malignancy (21). A follow up study done in England among 163 asymptomatic patients with elevated levels of TSH who were also positive for thyroid antibodies (anti-TPO, antiTgAbs) showed that these patients developed overt hypothyroidism at the rate of $5 \%$ per annum (22).

Markers of autoimmune thyroid disease are prevalent among Kenyan patients with primary thyroid disorders and the positivity of anti-TgAbs is higher than earlier reported. This study was however limited by the small sample size, and it did not include healthy subjects. The specificity of the thyroid autoimmune markers was therefore not evaluated. Validation of these findings is needed using a wider study, including both healthy individuals and patients with thyroid disorders. 


\section{REFERENCES}

1. Volpe, R. Autoimmune Thyroiditis: In:The Thyroid-A fundamental and a clinical text. Lewis, E. B. and Utiger, R. D. $6^{\text {th }}$ ed. 1991: J.B Lippincott Co (ed). pg. 921-933.

2. Hashimoto, H. Struma lymphomatosa. Arch. Clin. Chem. 1912; 97: 219-220.

3. Hollowell, G.J., Norman, W.S.,Hannon, W.H., et al. Serum TSH, T4 and Thyroid antibodies in the United States population (1988 to 1994): National Health and Nutrition Examination Survey (NHANES III). J. Clin. Endocrinol. \& Metabolism. 2002; 87: 489-499.

4. Hassanat, M. A., Runi, M. A., Alam, M. N. and Salam, M.A. Status of antithyroid antibodies in Bangladesh. Postgrad. Med. J. 2000; 76: 345-349.

5. Bowry, T. R. and Radia, R. Thyroid autoantibody profile in African thyrotoxicosis. East. Afr. Med. J. 1979; 56: 640-645.

6. Okosieme, O.E., Taylor, R.C., Ohwovoriole, A.E., et al. Prevalence of thyroid antibodies in Nigerian patients. Q. J. Med. 2007; 100: 107-112.

7. Laurberg, P., Pedersen, K.M., Hreidarsson, A., et al. Iodine intake and the pattern of thyroid disordersA comparative epidemiological study of thyroid abnormalities in the elderly in Iceland and in Jutland, Denmark. J. Clin. Endocrinol. \& Metab. 1998; 83: 765-769.

8. Doufas, A.G., Mastorakos, G., Chatziioannou, S., et al The predominant form of non-toxic goitre in Greece is now autoimmune thyroiditis. Eur. J. Endocrinol. 1999; 40: 505-511.

9. Government of Kenya. Food, Drugs and Chemical substances Act, Laws of Kenya. 1992. 254: 299.

10. Mc Gregor, A.M. The thyroid gland and disorders of thyroid function. In: Oxford Textbook of Medicine. Weatherhall, D., Lendingham, J. and Warell, D.A. $3^{\text {rd }}$ ed. 1996:Oxford. (ed). 1603-1621

11. Kast, R. E. Predominance of autoimmune and rheumatic disease in females. J. Rheumatol. 1977; 4: 288-292.
12. Mahmoud, S., Islam, M.M., Siddiqui, N.I., et al. Prevalence of antithyroid antimicrosomal antibodies in thyroid patients of endemicgoitre area. Mymensingh Med. J. 2004; 13: 4-10.

13. Mc Gill, P.F. Thyrotoxicosis in the Africans: clinical and immunological observations. Brit. J. Med. 1971; 2: 679-670.

14. Weetman, A.P. and Mc Gregor, A.M. Autoimmune thyroid disease: Developments in our understanding. Endocr. Rev. 1984; 5: 309-350.

15. Nye, L., Roit ,I. M. and Decarvalho ,L.P. An investigation of the clonality of human autoimmune thyroglobulin antibodies and their light chains. Clin. Exp. Immunol. 1981; 46:12.

16. Volpe', R. Antibodies to thyroid disease. In: The Thyroid- A Fundamental and a Clinical Text. Lewis, E. B. and Utiger, R. D. $6^{\text {th }}$ ed. 1991: J. B. Lippincott Co. (ed). p. 506-523. Philadephia.

17. Naito, N. and Saito, K. Antithyroglobulin antibodies in sera from patients with chronic thyroiditis and from healthy subjects-differences in cross-reactivity with thyroid peroxidase. Clin. Exp. Immunol.1990; 80: 4-5.

18. Tietz, N. W. Clinical Guide to Laboratory Tests. $3^{\text {rd }}$ ed.1995: Saunders (ed).p.589.

19. Jannson, R., Karlson, A. and Dahlberg, P.A. Thyroxine, Methimazole and thyroid antimicrosomal antibodies in Hashimoto's thyroiditis. Brit. J. Med. 1985; 290: 11-13.

20. Irvine, W.J. and Stuart, A. G. Prognostic significance of thyroid antibodies in the management of thyrotoxicosis. E\&S Livingstone Ltd (ed). 1961;London. p 118.

21. Ginsberg, J.Diagnosis and management of Graves disease. CMAJ. 2003; 160: 5.

22. Turnbridge, W. G., Brown, M. and Freud, J. M. Natural history of autoimmune thyroiditis. Brit. J. Med. 1981; 282: 258-262. 